\title{
Determination of the spectral dependence for the absorption coefficient of phosphor inorganic microparticles
}

\author{
D.N. Khmil’, A.M. Kamuz, P.F. Oleksenko, V.G. Kamuz, N.G. Aleksenko, O.A. Kamuz \\ $V$. Lashkaryov Institute of Semiconductor Physics, NAS of Ukraine, 03028 Kyiv, Ukraine \\ Phone: +38(044)525-6168,e-mail:deniskhmil@ukr.net,kamuz_a_m@ukr.net
}

\begin{abstract}
Developed in the work has been the mathematical model of the composite film, which enables to calculate the wavelength dependence of the absorption coefficient of microcrystals in the phosphor composition. Also, developed has been a new method for determining the spectrum of the absorption coefficient for microparticles of powderlike materials. Being based on this method, obtained has been the wavelength dependence for the absorption coefficient of microparticles of inorganic phosphor ФЛЖ-7-01. At the emission peak of a blue LED $(456 \mathrm{~nm})$ their absorption coefficient is close to $684 \mathrm{~cm}^{-1}$.
\end{abstract}

Keywords: absorption coefficient, phosphor, photoluminophore, light scattering, white LED.

Manuscript received 16.04.15; revised version received 21.07.15; accepted for publication 03.09.15; published online 30.09.15.

\section{Introduction}

Up to date, facilities and universal methods capable to provide measurements of the absorption coefficient of powder materials (for example, powder phosphors (photoluminophore, luminofor) for white LEDs) are absent. Therefore, both the theory of these substances and their practical application are based exclusively on investigations of luminescent emission [1-7], diffuse reflection [8-12] and transmission spectra [13], excitation spectra [14-18] as well as on the absorption ones [19]. Since these investigations cannot provide separated registration of the parts of the scattered and absorbed light, it is not possible to calculate the main optical characteristic of powder materials - their absorption coefficient. It means that there is no possibility to calculate values of such important characteristics of powder-like phosphor as: quantum efficiency, optimal concentration of the activator inside microparticles, and so on. Therefore, when developing new luminophors, researchers use the only one method trial-and-error procedure. This, consequently, makes the technology to be an expensive and long process.

Earlier, we demonstrated for the first time a new method to determine the spectrum of absorption coefficient of the films made of phosphor suspension [20]. In this work, we developed the new method for determination of the absorption coefficient spectrum inherent to microparticles of powder-like materials, which is based on a mathematical model of the film prepared from the phosphor suspension, and their absorption coefficient spectra were obtained using the "method of two thicknesses". Shown in this paper [20] is also the absorption coefficient spectrum of the inorganic phosphor ФЛЖ-7-01, which was obtained for the first time by using the new method. 


\section{Development of the model}

In the paper [20], we described the simple "method of two thicknesses" that allows quantitative calculation of the absorption coefficient spectrum for a composite film prepared from the phosphor suspension. It is clear that the spectrum depends on the phosphor concentration in this suspension, distribution of microparticles by their dimensions as well as on the concentration of activator in each particle.

Since the spectrum of the absorption coefficient of the composite film (by using the method of two thicknesses), and the concentration and sizes of microparticles in it are known, then to define the absorption coefficient spectrum of phosphor microcrystals, at first we have developed a corresponding mathematical model of the composite film. Then, we have determined a quantitative relation between the known coefficient of film absorption and the absorption coefficient of the phosphor microcrystals.

The model of the film prepared from the phosphor suspension can be developed in the following way. Let us choose the part of the film where the light intensity decreases only due to absorption [20] and cut out from it the rectangular parallelepiped with the height equal to $H$ and the base in the form of a square with the area equal to $S$ (see Fig. 1). Separate this parallelepiped by planes parallel to the base by the layers $P_{1}, P_{2}, P_{3}, \ldots, P_{N}$ with the thickness equal to the mean size $a$ of phosphor microparticles. Separate the layer $P_{1}$ by unit cells with the base area $s_{0}=a^{2}$. Let us assume that the amount of unit cells in this layer is equal to $M$ (it is obvious that $S=s_{0} \cdot M$ ) and each particle of the phosphor powder has a cubic shape and its volume equal to that of unit cell.

Let the amount of cubic particles in the layer $P_{1}$ is $n(n<M)$. The sums of areas for all the particles and empty cells (non-filled with phosphor particles) in the layer $P_{1}$ are, respectively:

$S_{P}^{(1)}=s_{0} \cdot n$

and

$S_{E}^{(1)}=s_{0} \cdot(M-n)$.

Then, the area of the layer $P_{1}$ is

$S^{(1)}=S_{P}^{(1)}+S_{E}^{(1)}$.

If the microparticle concentration in the layer $P_{1}$ is equal to $K$, then

$K=\left(\frac{S_{P}}{S}\right)=\frac{s_{0} \cdot n}{s_{0} \cdot M}=\frac{n}{M}$

and the concentration of empty cells

$\frac{S_{E}}{S}=\frac{s_{0} \cdot(M-n)}{s_{0} \cdot M}=\frac{M-n}{M}=1-K[21]$.

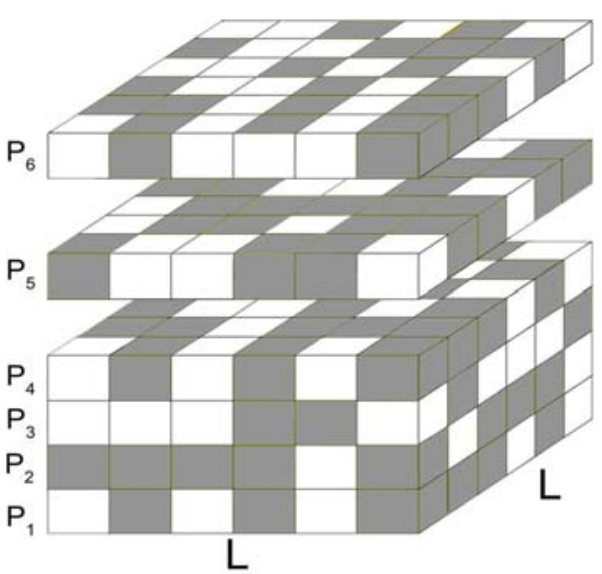

Fig. 1. Scheme of the six-layer film prepared from phosphor suspension.

Since the microparticles in this parallelepiped are chaotically distributed, it is clear that in every another layer $P_{2}, \ldots, P_{N}$ the sums of areas for all the particles and empty cells have the same values:

$S_{P}^{(1)}=S_{P}^{(2)}=\ldots=S_{P}^{(n)}=s_{0} \cdot n$

and

$S_{E}^{(1)}=S_{E}^{(2)}=\ldots=S_{E}^{(n)}=s_{0} \cdot(M-n)$.

If the parallelepiped is separated by $N$ layers, then each layer contains $N$ areas of particles and $(M-n)$ areas of empty cells. Let us make a projection of the areas of micro-particles and empty cells for all $N$ layers onto the area of the parallelepiped base and find the areas of overlapping projections for areas of particles and empty cells for all $N$ layers in the following way.

Let the light beam falls onto the layer $P_{1}$ along the normal to it (in Fig. 1, light propagates from the bottom to the top), the light flux is $I$ and its surface density $-\frac{I}{S}$. It is clear from the geometrical definition of probability that the ratio $\frac{S_{E}^{(1)}}{S}$ corresponds to the probability of propagation of the light beam beside the microparticle (i.e., it defines the part of the beam that is not absorbed by particles), and $\frac{S_{E}^{(1)}}{S}$ corresponds to the probability for the plane-parallel beam to fall onto the particle (i.e., it defines the part of the light beam that can be absorbed by the particle). Diffraction of light is not taken into account in this consideration. Then, with account of the said above, the projections of empty cell areas and phosphor microparticles for the single layer $P_{1}$ consist of two parts:

$S_{E}^{(1)}=(1-K) \cdot S$, 
$S_{P}^{(1)}=K \cdot S$

Let us assume that empty cells do not absorb light. Then, using the Bouger-Lambert law one can write expressions for radiation fluxes that pass the first $\left(S_{E}^{(1)}\right)$ and second $\left(S_{P}^{(1)}\right)$ projective planes:

$I_{E}=\frac{I}{S}[(1-K) \cdot S]=I \cdot(1-K)$,

$I_{P}=\frac{I}{S}[K \cdot S] \cdot e^{-\beta_{\lambda} \cdot a}=I \cdot K \cdot e^{-\beta_{\lambda} \cdot a}$,

where $\beta_{\lambda}$ is the absorption coefficient for mean microparticles of phosphor $\left(d_{50}\right)$.

Being based on the equations (3), (10) and (11), one can write the equation for the radiation flux $I_{1}$ that comes out of the first layer of the film

$I_{1}=I_{E}+I_{P}=I \cdot(1-K)+I \cdot K \cdot e^{-\beta_{\lambda} \cdot a}$.

Let us divide the equation (12) by $I$ :

$\frac{I_{1}}{I}=1-K+K \cdot e^{-\beta_{\lambda} \cdot a}$,

Then with account of the fact that for the single layer of the film $\frac{I_{1}}{I}=e^{-\alpha_{\lambda} \cdot a}$, one can write $e^{-\alpha_{\lambda} \cdot a}=1-K+K \cdot e^{-\beta_{\lambda} \cdot a}$

In the left and right parts of the equation (14), we used the parameters of the single film layer and phosphor microparticles, respectively, namely: $\alpha_{\lambda}$ is the absorption coefficient for the film from suspension (determined using the method of two thicknesses) and $\beta_{\lambda}$ is the absorption coefficient for phosphor microparticles of mean sizes $\left(d_{50}\right)$.

Let us add the layer $P_{2}$ to the layer $P_{1}$ and find the probability of overlapping between projections of areas inherent to empty cells and those of phosphor microparticles in these layers, in other words, the probabilities of overlapping between areas $S_{\mathrm{E}}^{(2)}$ and $S_{E}^{(1)}, S_{E}^{(2)}$ and $S_{P}^{(1)}, S_{P}^{(2)}$ and $S_{P}^{(1)}, S_{P}^{(2)}$ and $S_{P}^{(1)}$ (superscript indicates the layer number). Since microparticles and empty cells in $P_{1}$ and $P_{2}$ layers are placed in a chaotic way, the probabilities of overlapping between these areas are equal to the product of the respective probabilities, namely:

$\frac{S_{E E}^{(2,1)}}{S}=\frac{S_{E}^{(2)}}{S} \cdot \frac{S_{E}^{(1)}}{S}$,
$\frac{S_{E P}^{(2,1)}}{S}=\frac{S_{E}^{(2)}}{S} \cdot \frac{S_{P}^{(1)}}{S}$,
$\frac{S_{P E}^{(2,1)}}{S}=\frac{S_{P}^{(2)}}{S} \cdot \frac{S_{E}^{(1)}}{S}$,
$\frac{S_{P P}^{(2,1)}}{S}=\frac{S_{P}^{(2)}}{S} \cdot \frac{S_{P}^{(1)}}{S}$, where $\frac{S_{E E}^{(2,1)}}{S}$ is the probability of overlapping between $\operatorname{areas} S_{\mathrm{E}}^{(2)}$ and $S_{\mathrm{E}}^{(1)} ; \frac{S_{E P}^{(2,1)}}{S}-$ respective probability for $S_{\mathrm{E}}^{(2)}$ and $S_{\mathrm{P}}^{(1)} ; \frac{S_{P E}^{(2,1)}}{S}-$ for $S_{\mathrm{P}}^{(2)}$ and $S_{\mathrm{E}}^{(1)} ; \frac{S_{P P}^{(2,1)}}{S}-$ for $S_{\mathrm{p}}{ }^{(2)}$ and $S_{\mathrm{P}}^{(1)}[21]$. Then, one can write:

$\frac{S_{E E}^{(2,1)}}{S}=(1-K)(1-K)=(1-K)^{2}$,

$\frac{S_{E P}^{(2,1)}}{S}=K \cdot(1-K)$,

$\frac{S_{P E}^{(2,1)}}{S}=K \cdot(1-K)$,

$\frac{S_{P P}^{(2,1)}}{S}=K \cdot K=K^{2}$

To determine the areas of overlapping, the respective probability should be multiplied by the total area $S$ :

$S_{E E}^{(2,1)}=(1-K)^{2} \cdot S$,

$S_{E P}^{(2,1)}=(1-K) \cdot K \cdot S$,

$S_{P E}^{(2,1)}=(1-K) \cdot K \cdot S$,

$S_{P P}^{(2,1)}=K^{2} \cdot S$.

As seen, projections of the areas of empty cells and phosphor microparticles for the layer $P_{1}$ consist of two parts $S_{E}^{(1)}$ and $S_{P}^{(1)}$, while for two layers $\left(P_{1}\right.$ and $\left.P_{2}\right)$ consist of four parts $S_{E E}^{(2,1)}, S_{E P}^{(2,1)}, S_{P E}^{(2,1)}$ and $S_{P P}^{(2,1)}$.

As it follows from the equations (24) and (25), $S_{E P}^{(2,1)}=S_{P E}^{(2,1)}=(1-K) \cdot K \cdot S, \quad$ therefore the total projection area from two layers can be expressed as follows:

$$
\begin{aligned}
& S=S_{E E}^{(2,1)}+S_{P E}^{(2,1)}+S_{E P}^{(2,1)}+S_{P P}^{(2,1)}=(1-K)^{2} \cdot S+ \\
& +(1-K) \cdot K \cdot S+(1-K) \cdot K \cdot S+K^{2} \cdot S= \\
& =S \cdot\left[(1-K)^{2}+2 K \cdot(1-K)+K^{2}\right] .
\end{aligned}
$$

Having analyzed the equation (27), one can see that, after summation of $P_{2}$ and $P_{1}$ layers, the planeparallel light beam passes through three planes, areas of which are equal to $(1-K)^{2} \cdot S, 2 K \cdot S \cdot(1-K)$ and $K^{2} \cdot S$, respectively. In other words, parts of the light beam will pass through two empty cells (with the area $\left.(1-K)^{2} \cdot S\right)$, through one empty cell and one microparticles (with the area $2 K \cdot S \cdot(1-K))$ as well as through two microparticles (area $K^{2} \cdot S$ ). Then, the light flux passing through the first, second and third will be, respectively, equal to: 
$I_{E E}=\frac{I}{S}\left[(1-K)^{2} \cdot S\right]=I \cdot(1-K)^{2}$,

$I_{E P}=\frac{I}{S}[2 K \cdot S \cdot(1-K)] \cdot e^{-\beta_{\lambda} \cdot a}=$

$=I \cdot 2 K \cdot(1-K) \cdot e^{-\beta_{\lambda} \cdot a}$,

$I_{P P}=\frac{I}{S}\left[K^{2} \cdot S\right] \cdot e^{-2 \beta_{\lambda} \cdot a}=I_{0} \cdot K^{2} \cdot e^{-2 \beta_{\lambda} \cdot a}$.

Being based on the equations (27) - (30), one can write the equation for the radiation flux $I_{2}$ that comes out of two film layers:

$$
\begin{aligned}
& I_{2}=I \cdot(1-K)^{2}+I \cdot 2 K \cdot(1-K) \cdot e^{-\beta_{\lambda} \cdot a} \\
& +I \cdot K^{2} \cdot e^{-2 \beta_{\lambda} \cdot a} .
\end{aligned}
$$

Dividing the equation (31) by $I$, one can obtain

$\frac{I_{2}}{I}=(1-K)^{2}+2 K \cdot(1-K) \cdot e^{-\beta_{\lambda} \cdot a}+$

$+K^{2} \cdot e^{-2 \beta_{\lambda} \cdot a}$.

Having taken into account that for two layers of the film $\frac{I_{2}}{I}=a^{-\alpha \lambda \cdot 2 \cdot a}$, it can be written

$$
\begin{aligned}
& e^{-\alpha_{\lambda} \cdot 2 \cdot a}=(1-K)^{2}+2 K \cdot(1-K) \cdot e^{-\beta_{\lambda} \cdot a} \\
& +K^{2} \cdot e^{-2 \beta_{\lambda} \cdot a} .
\end{aligned}
$$

In the left and right parts of Eq. (33), we used parameters of two film layers and of phosphor particles, respectively.

If two layers $\left(P_{1}\right.$ and $\left.P_{2}\right)$ are added with the third one that consists of two areas $\left(S_{E}^{(3)}\right.$ and $\left.S_{P}^{(3)}\right)$, then these areas of the third layer will be overlapped with four projections of areas corresponding to the layers $P_{1}$ and $P_{2}$. It means that the probabilities of overlapping between the areas $S_{E}^{(3)}$ and $S_{P}^{(3)}$ with projections of the areas $S_{E E}^{(2,1)}, S_{E P}^{(2,1)}, S_{E P}^{(2,1)}$ and $S_{P P}^{(2,1)}$ will be, respectively, equal to

$$
\begin{aligned}
& \frac{S_{E E E}^{(321)}}{S}=\frac{S_{E}^{(3)}}{S} \cdot \frac{S_{E E}^{(21)}}{S}= \\
& =(1-K) \cdot(1-K)^{2}=(1-K)^{3}, \\
& \frac{S_{E E P}^{(321)}}{S}=\frac{S_{E}^{(3)}}{S} \cdot \frac{S_{E P}^{(21)}}{S}= \\
& =(1-K) \cdot(1-K) \cdot K=K \cdot(1-K)^{2}, \\
& \frac{S_{E P E}^{(321)}}{S}=\frac{S_{E}^{(3)}}{S} \cdot \frac{S_{E P}^{(21)}}{S}= \\
& =(1-K) \cdot(1-K) \cdot K=K \cdot(1-K)^{2}, \\
& \frac{S_{E P P}^{(321)}}{S}=\frac{S_{E}^{(3)}}{S} \cdot \frac{S_{P P}^{(21)}}{S}=K^{2} \cdot(1-K),
\end{aligned}
$$

$$
\begin{aligned}
& \frac{S_{P E E}^{(321)}}{S}=\frac{S_{P}^{(3)}}{S} \cdot \frac{S_{E E}^{(21)}}{S}=K \cdot(1-K)^{2}, \\
& \frac{S_{P E P}^{(321)}}{S}=\frac{S_{P}^{(3)}}{S} \cdot \frac{S_{E P}^{(21)}}{S}=K^{2} \cdot(1-K), \\
& \frac{S_{P P E}^{(321)}}{S}=\frac{S_{P}^{(3)}}{S} \cdot \frac{S_{P E}^{(21)}}{S}=K^{2} \cdot(1-K), \\
& \frac{S_{P P P}^{(321)}}{S}=\frac{S_{P}^{(3)}}{S} \cdot \frac{S_{P P}^{(21)}}{S}=K \cdot K^{2}=K^{3} .
\end{aligned}
$$

It follows from the equations (35), (36), (38) and (37), (39), (40) that

$S_{E E P}^{(321)}=S_{E P E}^{(321)}=S_{P E E}^{(321)}=S \cdot\left[K \cdot(1-K)^{2}\right]$,

and

$S_{E P P}^{(321)}=S_{P E P}^{(321)}=S_{P P E}^{(321)}=S \cdot\left[K^{2} \cdot(1-K)\right]$.

Then, with account of the equations (3) and (34) (43) the total projection area of three layers can be expressed as follows:

$$
\begin{aligned}
& S=S_{E E E}^{(321)}+S_{E E P}^{(321)}+S_{E P E}^{(321)}+S_{P E E}^{(321)}+ \\
& +S_{E P P}^{(321)}+S_{P E P}^{(321)}+S_{P P E}^{(321)}+S_{P P P}^{(321)}= \\
& =S \cdot\left[(1-K)^{3}+3 K \cdot(1-K)^{2}+3 K \cdot(1-K)+K^{3}\right] .
\end{aligned}
$$

After addition of the layer $P_{3}$ to the layers $P_{2}$ and $P_{1}$, the plane-parallel light beam will pass through four areas different by their value that are equal, respectively, to $S \cdot(1-K)^{3}, S \cdot 3 K \cdot(1-K)^{2}, S \cdot 3 K \cdot(1-K)$ and $S \cdot K^{3}[21]$. In this case, light passes through three empty cells (area $S \cdot(1-K)^{3}$ ), through two empty cells and one phosphor microparticle (area $S \cdot 3 K \cdot(1-K)^{2}$ ), through one empty cell and one microparticle (area $S \cdot 3 K \cdot(1-K))$ as well as through three microparticles (area $S \cdot K^{3}$ ).

Starting from the said above, one can state that through the first, second, third and fourth parts the respective radiation fluxes will pass

$$
\begin{aligned}
& I_{E E E}=\frac{I}{S}\left[S \cdot(1-K)^{3}\right]=I \cdot(1-K)^{3}, \\
& I_{E E P}=\frac{I}{S}\left[S \cdot 3 K \cdot(1-K)^{2}\right] \cdot e^{-\beta_{\lambda} \cdot a}= \\
& =I \cdot 3 K \cdot(1-K)^{2} \cdot e^{-\beta_{\lambda} \cdot a}, \\
& I_{E P P}=\frac{I}{S}\left[S \cdot 3 K^{2} \cdot(1-K)\right] \cdot e^{-2 \beta_{\lambda} \cdot a}= \\
& =I \cdot 3 K^{2} \cdot(1-K) \cdot e^{-2 \beta_{\lambda} \cdot a}, \\
& I_{P P P}=\frac{I}{S}\left[S \cdot K^{3}\right] \cdot e^{-3 \beta_{\lambda} \cdot a}=I \cdot K^{3} \cdot e^{-3 \beta_{\lambda} \cdot a} .
\end{aligned}
$$

Being based on the equations (44) to (48), one can write an equation for the radiation flux $I_{3}$ that goes out of the three layers in the film of phosphor 
$I_{3}=I \cdot(1-K)+I \cdot 3 K \cdot(1-K)^{2} \cdot e^{-\beta_{\lambda} \cdot a}+$

$+I \cdot 3 K^{2} \cdot(1-K) \cdot e^{-2 \beta_{\lambda} \cdot a}+I \cdot K^{3} \cdot e^{-3 \beta_{\lambda} \cdot a}$.

Dividing Eq. (49) by $I$, one can obtain

$\frac{I_{3}}{I}=(1-K)+3 K \cdot(1-K)^{2} \cdot e^{-\beta_{\lambda} \cdot a}+$

$+3 K^{2} \cdot(1-K) \cdot e^{-2 \beta_{\lambda} \cdot a}+K^{3} \cdot e^{-3 \beta_{\lambda} \cdot a}$.

With account of the fact that for three film layers $\frac{I_{3}}{I}=e^{-\alpha_{\lambda} \cdot 3 \cdot a}$, it should be written

$e^{-\alpha_{\lambda} \cdot 3 \cdot a}=(1-K)^{3}+3 K \cdot(1-K)^{2} \cdot e^{-\beta_{\lambda} \cdot a}+$

$+3 K^{2} \cdot(1-K) \cdot e^{-2 \beta_{\lambda} \cdot a}+K^{3} \cdot e^{-3 \beta_{\lambda} \cdot a}$.

In the left and right parts of Eq. (51), we used the parameters of three film layers and phosphor particles, respectively.

Using analogous considerations, we determined overlapping of area projections for empty cells and microparticles from 4, 5, 6 and so on layers. Then, we deduced a mnemonic rule to determine the probability of overlapping between projection areas from $N$ layers.

The developed mathematical model of the composite film was applied to determine the dependence of absorption coefficients on the light wavelength for microcrystals $\left(d_{50}\right)$ available in inorganic phosphor ФЛЖ-7-01. The mean size of these microcrystals was $5 \mu \mathrm{m}$. For investigations, we used the 6-layer model described by the following equation

$$
\begin{aligned}
& e^{-\alpha_{\lambda} 6 a}=(1-K)^{6}+6 K(1-K)^{5} \cdot e^{-\beta_{\lambda} a}+ \\
& +15 K^{2}(1-K)^{4} \cdot e^{-2 \beta_{\lambda} \cdot a}+20 K^{3}(1-K)^{3} \cdot e^{-3 \beta_{\lambda} a}+ \\
& +15 K^{4}(1-K)^{3} \cdot e^{-4 \beta_{\lambda} a}+6 K^{5}(1-K) \cdot e^{-5 \beta_{\lambda} a}+ \\
& +K^{6} \cdot e^{-6 \beta_{\lambda} a} .
\end{aligned}
$$

It should be noted that the probability of overlapping between projection areas depends on the concentration of microparticles. See, for example, Fig. 2 where adduced are diagrams of overlapping to projection areas in the 6-layer film for the microparticle concentrations 30 and $60 \%$.

Note that, in the above Eq. (52) for the 6-layer film, the value $(1-K)^{6}$ is equal to projection areas only from empty cells, while the values $6 K \cdot(1-K)^{5}$, $15 K^{2} \cdot(1-K)^{4}, 20 K^{3} \cdot(1-K)^{3}, 6 K^{5} \cdot(1-K)$ and $K^{6}$ are equal to the projection areas of overlapping, which arise from one, two, three, four, five and six photoluminophore particles, respectively.

To use this equation, first it is necessary to determine the absorption coefficient $\alpha_{\lambda}$ of the composite film prepared from phosphor suspension (applying the method of two thicknesses) and then to use the equation (52) or some similar one, with account of the amount of layers. After that, one should perform calculation of the absorption coefficient for microcrystals $\beta_{\lambda}$. It is noteworthy that the left part of Eq. (52) contains parameters of the film that consists of 6 layers, while the right one - the values of 7 partial radiation fluxes that pass through 6 overlapping layers:

- $(1-K)^{6}$ - for 6 empty cells,

- $6 K \cdot(1-K)^{5}$ - for 5 empty cells and one cell containing a microcrystal of phosphor,

- $15 K^{2} \cdot(1-K)^{4}-$ for 4 empty cells and 2 cells with microcrystals,

- $20 K^{3} \cdot(1-K)^{3}$ - for 3 empty cells and 3 cells with microcrystals,

- $\quad 15 K^{4} \cdot(1-K)^{2}-$ for 2 empty cells and 4 cells with microcrystals,

- $6 K^{5} \cdot(1-K)$ - for one empty cell and 5 cells with microcrystals,

- $K^{6}$ - for 6 cells with microcrystals.
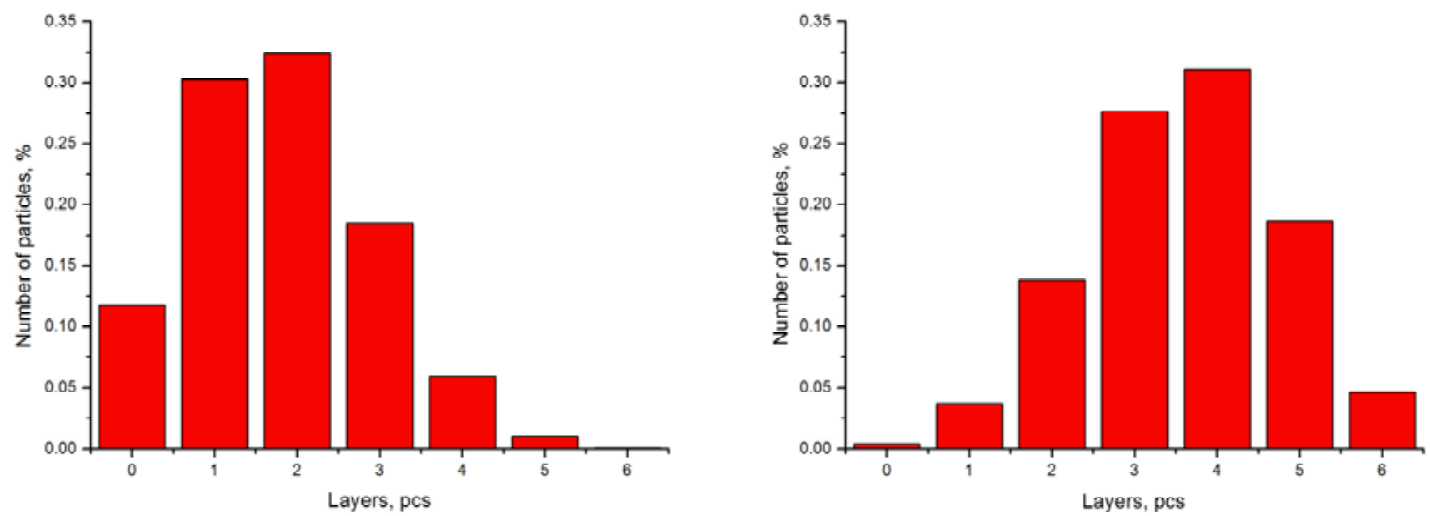

Fig. 2. The probability of overlapping phosphor particles in six-layer film with the phosphor concentration: $a-30 \%, b-60 \%$. 


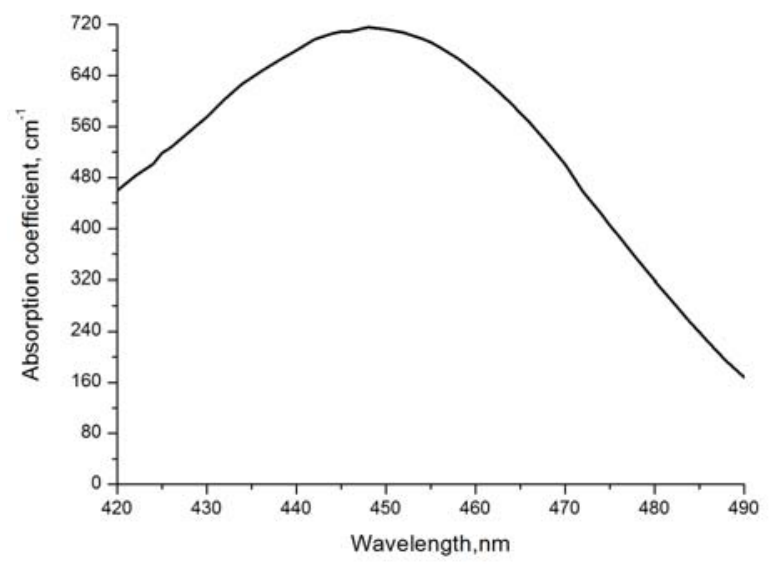

Fig. 3. Wavelength dependence of the absorption coefficient for phosphor microcrystals of mean sizes $\left(d_{50}=5 \mu \mathrm{m}\right)$.

\section{Results of experiment}

Using the developed method, we plotted the dependence of the absorption coefficient on the light wavelength for microcrystals immersed in the film prepared from phosphor suspension. Fig. 3 shows the calculated plot for the wavelength dependence of the absorption coefficient inherent to phosphor microcrystals of mean sizes $\left(d_{50}\right)$. The absorption coefficient for the emission peak of the blue LED $(456 \mathrm{~nm})$ is equal to $684 \mathrm{~cm}^{-1}$, while the absorption peak of the mean microcrystals is located near $448 \mathrm{~nm}$ and equal to $716 \mathrm{~cm}^{-1}$.

\section{Conclusions}

1. Developed in the work has been the mathematical model of the composite film, which enables to calculate the wavelength dependence of the absorption coefficient inherent to phosphor microcrystals.

2. Also, developed has been a new method for determining the spectrum of the absorption coefficient for microparticles of powder-like materials.

Being based on this method, obtained has been the wavelength dependence for the absorption coefficient of microparticles of inorganic phosphor ФЛЖ-7-01. At the emission peak of a blue LED (456 nm) their absorption coefficient is close to $684 \mathrm{~cm}^{-1}$.

\section{References}

1. Manisha Upasani, Bhavana Butey, S.V. Moharil, Luminescence studies on lanthanide ions $\left(\mathrm{Gd}^{3+}\right.$, $\mathrm{Tb}^{3+}$ ) doped YAG:Ce phosphors by combustion synthesis // J. Appl. Phys. 6(2), p. 28-33.

2. Arunachalam Lakshmanan, R Satheesh Kumar, V. Sivakumar, Preema C. Thomas, M.T. Jose, Synthesis, photoluminescence and thermal quenching of YAG:Ce phosphor for white light emitting diodes // Indian J. Pure \& Appl. Phys. 49, p. 303-307 (2011).

3. Min Yeong Kim, Dong-Sik Bae, Photoluminescence of $\mathrm{Y}_{3}(\mathrm{Al}, \mathrm{Ga})_{5} \mathrm{O}_{12}: \mathrm{Ce}^{3+}$ nanoparticles by a reverse micelle process // Kor. J. Mater. Res. 23(1), p. 31-34 (2013).

4. Zhiguo Xia, Daimei Chen, Min Yang, Ting Ying, Synthesis and luminescence properties of $\mathrm{YVO}_{4}: \mathrm{Eu}^{3+}, \mathrm{Bi}^{3+}$ phosphor with enhanced photoluminescence by $\mathrm{Bi}^{3+}$ doping // J. Phys. Chem. Solids, 71, p. 175-180 (2010).

5. Zhiguo Xia, Jiaqing Zhuang, Libing Liao, Haikun Liu, Yi Luo, and Peng Du, Synthesis and luminescence properties of $\mathrm{Ba}_{2} \mathrm{Gd}\left(\mathrm{BO}_{3}\right)_{2} \mathrm{Cl}: \mathrm{Eu}^{2+}$ phosphor // J. Electrochem. Soc. 158(11), p. J359J362 (2011).

6. Huijuan Yang, Guisheng Zhu, Le Yuan, Chi Zhang, Fengsheng Li, Huarui $\mathrm{Xu}$ and Aibing $\mathrm{Yu}$, Characterization and luminescence properties of YAG: $\mathrm{Ce}^{3+}$ phosphors by molten salt synthesis // J. Am. Ceram. Soc. 95(1), p. 49-51 (2012).

7. V. Makhov, Khaidukov, Luminescence of YAG doped with $\mathrm{Eu}, \mathrm{Yb}$, and $\mathrm{Mn}$ ions under VUV excitation // Opt. Spectrosc. 116(5), p. 748 (2014).

8. Ru-Shi Liu, Yu-Huan Liu, Nitin C. Bagkar, ShuFen $\mathrm{Hu}$, Enhanced luminescence of $\mathrm{SrSi}_{2} \mathrm{O}_{2} \mathrm{~N}_{2}$ $: \mathrm{Eu}^{2+}$ phosphors by codoping with $\mathrm{Ce}^{3+}, \mathrm{Mn}^{2+}$, and $\mathrm{Dy}^{3+}$ ions // Appl. Phys. Lett. 91, p. 061119 (2007).

9. D. Michalik, J. Plewa, M. Sopicka-Lizer, T. Justel, Cerium doped yttrium aluminium garnet modified by silicon and nitrogen // Ceramic Materials, 60(4), p. 225-228 (2008).

10. Jin An Jeong, Kyunghwan Park, Dong Hoon Lee, Hong Gun Kim, Yoo Young Kim, The characteristics of YAG:Ce phosphor powder prepared using a $\mathrm{NO}^{3-}$-malonic acid- $\mathrm{NH}_{4} \mathrm{NO}_{3}-$ $\mathrm{NH}_{3} \cdot \mathrm{H}_{2} \mathrm{O}$ system // Bull. Korean Chem. Soc. 33(4), p. 1141-1146 (2012).

11. D. Michalick, M. Sopicka-Lizer, J. Plewa, T. Pawlik, Application of mechanochemical processing to synthesis of YAG:Ce garnet powder // Archives of Metallurgy and Materials, 56(4), p. 1257-1264 (2011).

12. R.L.P. van Veen, W. Verkruysse, and H.J.C. M. Sterenborg, Diffuse-reflectance spectroscopy from 500 to $1060 \mathrm{~nm}$ by correction for inhomogeneously distributed absorbers // Opt. Lett. 27(4), p. 246-248 (2002).

13. S. Nishiura, S. Tanabe, K. Fujioka, Y. Fujimoto, M. Nakatsuka, Preparation and optical properties of transparent Ce:YAG ceramics for high power white LED // IOP Conf. Series: Materials Science and Engineering, 1, 012031 (2009).

14. Charusheela Joshi, Pooja Yadav, S.V. Moharil, Improved white light emitting diode characteristics by coating GdAG:Ce phosphor // Transaction on Electrical and Electronic Materials, 15(2), p. 69-72 (2014). 
15. V. Lojpur, A. Egelja, J. Pantić, V. Đorđević, B. Matović, M.D. Dramićanin, $\mathrm{Y}_{3} \mathrm{Al}_{5} \mathrm{O}_{12}: \mathrm{Re}^{3+}$ $(\mathrm{Re}=\mathrm{Ce}, \mathrm{Eu}$, and $\mathrm{Sm})$ nanocrystalline powders prepared by modified glycine combustion method // Science of Sintering, 46, p. 75-82 (2014).

16. M. Nazarov, Chulsoo Yoon, Chang Hoon Kwak, Jong Rak Sohn, Quantum efficiency of yttrium and terbium garnet // Moldavian J. Phys. Sci. 5(1), p. 42-49 (2006).

17. Yan Xing-huang, Zheng Song-sheng, Yu Rui-min, Cai Jing, Xu Zhi-wei, Liu Chun-jia, Luo Xue-tao, Preparation of YAG:Ce ${ }^{3+}$ phosphor by sol-gel low temperature combustion method and its luminescent properties // Trans. Nonferrous Met. Soc. China, 18, p. 648-653 (2008).

18. Ran Huang, Sai Li, Shaolin Xue , Zhixing Jiang, Shuxian $\mathrm{Wu}$, Preparation and characterization of YAG: $\mathrm{Ce}^{3+}$ phosphors by sol-solvothermal process
// 2012 International Conference on Future Environment and Energy IPCBEE, 28, p. 85-89 (2012).

19. Hsuan-Min Lee, Chao-Chi Cheng, ChiYuen Huang, The synthesis and optical property of solid-state-prepared YAG:Ce phosphor by a spraydrying method // Mater. Res. Bull. 44, p. 10811085 (2009).

20. D.N. Khmil, A.M. Kamuz, P.F. Oleksenko, V.G. Kamuz, N.G. Aleksenko, O.A. Kamuz, Method for determination of the absorption coefficient in films based on photoluminophor suspension for white LEDs // Semiconductor Physics, Quantum Electronics \& Optoelectronics 18(2), p. 215-219 (2015).

21. Jay L. Devore, Probability and Statistics for Engineering and the Sciences. Hardcover Textbook, 2011. 